ÉGYPTE

monde arabe

\section{Égypte/Monde arabe}

4 | 2007

Figures de la santé en Égypte

\title{
Le narguilé (chicha, hookah) : une épidémie venue d'Égypte?
}

\section{Kamal Chaouachi}

\section{(2) OpenEdition}

\section{Journals}

Édition électronique

URL : https://journals.openedition.org/ema/1776

DOI : 10.4000/ema.1776

ISSN : 2090-7273

\section{Éditeur}

CEDEJ - Centre d'études et de documentation économiques juridiques et sociales

\section{Édition imprimée}

Date de publication : 31 décembre 2007

Pagination : 247-267

ISBN : 2-6905838-43-4

ISSN : 1110-5097

\section{Référence électronique}

Kamal Chaouachi, «Le narguilé (chicha, hookah) : une épidémie venue d'Égypte ? », Égypte/Monde arabe [En ligne], 4 | 2007, mis en ligne le 31 décembre 2008, consulté le 07 juillet 2022. URL : http:// journals.openedition.org/ema/1776; DOI : https://doi.org/10.4000/ema.1776 
Kamal Chaouachi

\section{LE NARGUILÉ (CHICHA, HOOKAH) : UNE ÉPIDÉMIE MONDIALE VENUE D’ÉGYPTE?}

\section{LA MONDIALISATION DU NARGUILÉ}

I 'histoire et la médecine constituent aujourd'hui deux dimensions essen_tielles du narguilé. D'une part, les observateurs, quels qu'ils soient, posent systématiquement la question de l'origine de cet objet. D'autre part, ils s'interrogent tout autant sur les pathologies potentiellement associées à l'inhalation d'une fumée de tabac préalablement filtrée dans un récipient à eau et réputée moins nocive. À la croisée de ces deux champs, émerge la figure d'un médecin d'origine iranienne qui aurait, hypothèse historique, inventé ce dispositif alors qu'il était en fonction auprès de l'empereur Akbar dans I'Inde de l'an 1605. Quatre siècles se sont écoulés et cet objet traditionnel entre brusquement dans la modernité du troisième millénaire en interpellant notamment l'histoire de la médecine locale telle qu'elle s'écrit aujourd'hui. En effet, considéré jusquelà comme une pratique banale, voire «folklorique » de la vie quotidienne, le narguilé s'est vu transplanté aux quatre coins du monde et connaît, depuis une vingtaine d'années, un développement considérable, y compris dans son «berceau » moyen-oriental et tout particulièrement en Égypte. Un tel phénomène est déjà qualifié $d^{\prime}$ " épidémie » dans des études publiées dans des journaux biomédicaux internationaux. On verra plus loin si les modèles d'éradication et de prévention appliqués aux virus, aux maladies émergentes, aux drogues ou au tabagisme (par la cigarette) sont appropriés.

La pratique du narguilé était, il y a encore vingt ans, un fait qualifiable de social, culturel et historique. Depuis un peu moins de dix années, elle est devenue un fait sanitaire, économique et « global ». La convivialité particulière qui entoure cet usage - et qui renvoie à la sociabilité du monde arabo-islamique - n'est pas la moins remarquable des raisons de cette faveur. La marque égyptienne de tabamel ${ }^{1}$ Nakhla est ainsi devenue mondialement célèbre au cours de ces dix dernières années. Or, cette pratique s'est transplantée à la faveur des échanges liés au processus de mondialisation de la fin du siècle dernier et particulièrement du chassé-croisé des flux migratoires (Sud-Nord), 
particulièrement ceux liés à l'émigration égyptienne comme je le verrai plus loin, et au tourisme de masse (Nord-Sud), particulièrement autour du bassin méditerranéen, sans oublier ceux mentionnés par Karl Marx il y a cent vingt ans, ceux des marchandises. Ce dernier point n'est pas anodin quand on sait que la Chine fabrique et exporte désormais des narguilés.

La pratique du narguilé a pris rang d'épidémie alors qu'il n’y a pas si longtemps elle ne représentait qu'un usage traditionnel et sporadique de produits à base de tabac, qui n'avait apparemment pas posé de problème sanitaire majeur au cours des siècles passés. En fait, au Moyen-Orient et en Égypte en particulier, la région d'origine de cette pratique - pas nécessairement historique mais socioculturelle et quasi mythique du narguilé -, le renouveau a commencé il y a environ une vingtaine d'années et n'a pas faibli depuis. Mondialisation aidant, des va-et-vient ont eu lieu entre diverses parties du monde. Dans la région parisienne, on peut déjà recenser environ cent cinquante salons de type néo-orientaliste, tant par leur décoration que par leur raison commerciale : le narguilé. Les formes prises par ce phénomène sont semblables sur tous les continents et particulièrement aux États-Unis d'Amérique.

Ainsi, une réflexion scientifique sur ce sujet fait nécessairement partie de la problématique des figures de la santé en Égypte. Pourtant, il y a une dizaine d'années, cet objet apparemment anodin de la culture locale était tout à fait ignoré des biologistes. L'objet de recherche ${ }^{2}$ suscitait des regards, amusés, étonnés, voire méprisants.

Après une étude au Yémen (Chaouachi, 2001), je me suis tourné vers un terrain représenté par les sociétés autres qu'africaines ou asiatiques, ré-enchantées par l'éclosion des salons néo-orientalistes. Là, j'ai analysé les transformations de la sociabilité qu'implique la nouvelle pratique qui s'est exportée avec un certain succès commercial. L'hypothèse centrale de notre travail reposait sur une quadruple représentation collective et positive du phénomène : sa place privilégiée dans l'environnement socioculturel arabo-islamique, sa convivialité

1. Tabamel : préparation à base de tabac et de mélasse ou de miel destinée à être fumée dans le narguilé. Le nom arabe est mu'assil (mielleux, miellé). Le tabamel est disposé dans le fourneau (hagar, ra's) du narguilé (chîcha) et recouvert d'une feuille d'aluminium perforée (destinée à ralentir le chauffage) sur laquelle on place une pastille de charbon incandescent. On utilise parfois du tumbâk (tabac pur humidifié) à la place du tabamel ou encore du tabamel non aromatisé (comme celui de la célèbre marque égyptienne Zaghlûl). Ailleurs, ce produit est souvent appelé jurâk. II peut être utilisé indifféremment dans la moderne chicha ou la traditionnelle gûza (petit narguilé souvent individuel et portatif, dont le récipient à eau est une noix de coco évidée).

2. Suite à un premier livre (Chaouachi, 1997) proposant une synthèse des connaissances sur le sujet, nous avons poursuivi nos recherches sur le terrain, particulièrement au Yémen. 
spécifique, sa dimension sanitaire, son histoire ancienne et la réappropriation récente de cette dernière.

La socio-anthropologie, nouvelle discipline, s'intéresse aux conséquences épistémologiques du brouillage du proche et de l'éloigné ainsi que des « recouvrements d'univers, ceux du Même et l'Autre, domaines hier encore bien distincts " (Bouvier, 5164). Cependant, elle était insuffisante en raison de la dimension sanitaire qui est venue se superposer. Aussi, une formation à la tabacologie $\mathrm{e}^{3} \mathrm{~m}$ a permis de comprendre un certain

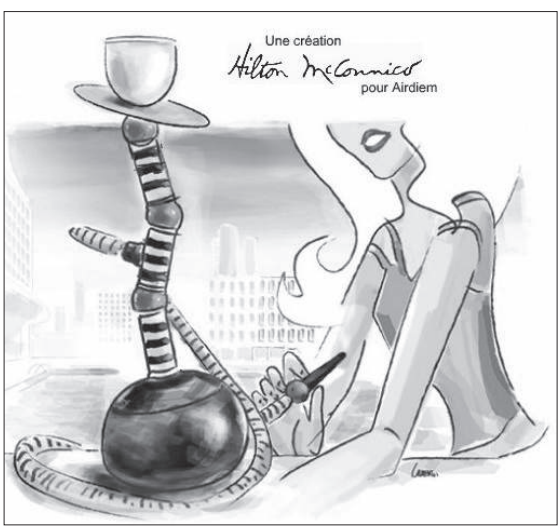

Le narguilé d'Airdiem pour les femmes parisiennes nombre de mécanismes complexes à l'œuvre dans l'usage du narguilé, d'autant plus utile que cette question a été, pour ainsi dire, prise en otage par la recherche biomédicale.

Les trois dernières années se sont caractérisées par une production abondante de publications s'efforçant de démontrer que le narguilé représente une catastrophe sanitaire. Les premières revues de synthèse des savoirs et de la littérature scientifique sur les effets du narguilé sur la santé m’ont frappé. Déconcerté par tant d'hypothétiques maladies et les innombrables effets nocifs dont le narguilé était soudain tenu pour responsable, j'ai d'abord été troublé de m'être intéressé à un objet aussi négatif. Allant directement aux sources originales de ces alarmes, j'ai découvert la pauvreté du langage des études biomédicales. Le réductionnisme, qui est supposé faire son efficacité, était aussi réducteur au plus haut point en présence d'une réalité aussi complexe que celle du narguilé. De fil en aiguille, j'ai découvert des biais méthodologiques et de graves erreurs. Il est impossible de faire l'impasse sur l'approche des sciences sociales à l'occasion du congrès mondial sur les défis sanitaires du xxle siècle (Chaouachi, 2005b).

Au passage, des biais relatifs aux représentations de l'Autre sautaient aux yeux. Certaines interdictions ne sont pas sans rapport avec le caractère « ethnique » et l'image " négative » de l'objet. Dès le début des années 1990, la Tunisie bannissait la pratique sur les terrasses des cafés. Dans l'esprit des responsables, les raisons relevaient probablement de la supposée mauvaise image que donneraient les habitants de ce pays aux millions de touristes qui le visitent chaque année. En Thaïlande, où vit une minorité musulmane souvent en conflit ouvert avec l'autorité centrale, la décision politique d'interdiction totale fut prise en 2003 en prenant pour caution scientifique l'un des articles alarmistes. En Israël,

3. Discipline qui traite des aspects médicaux, pharmacologiques, comportementaux, sociaux, culturels, historiques, économiques et autres, liés à l'usage du tabac. 
il y a deux ans, le ministère de la Défense invitait ses soldats à remplacer le narguilé par les bidis ${ }^{4}$, ce qui, d'un point de vue tabacologique, est aberrant, ces derniers produits semblant encore plus nocifs (goudrons) que les cigarettes. À Seattle (États-Unis), une récente mesure d'interdiction contre les indoor smoking (fumer en intérieur) vise directement les hookah bars. Un peu partout, dans ce pays, le développement des hookah lounges catalyse les nouvelles législations. Certains individus ou mouvements exploitent politiquement cette image " orientale ». On a vu ainsi des dirigeants de partis politiques d'extrême droite, Le Pen en France et Jirinovski en Russie, se faire photographier, voire interroger par des journalistes en fumant le narguilé. Pour le premier, il s'agissait d'un geste de défi à la classe politique française. Pour le second, à la veille de la première invasion américaine de l'Irak, la bravade visait à dénoncer la domination internationale du moment. En revanche, certains marchands surfent sur la vague actuelle et voudraient s'affranchir de la connotation orientale du narguilé. Ainsi, commence-t-il à revêtir, ça et là, des habits nouveaux, et des prototypes et formes nouvelles sont actuellement à l'étude, voire déjà sur le marché.

L'idée aujourd'hui prévaut que la dépendance tabagique est liée à la nicotine. Or, ce dogme, qui oriente la grande majorité des recherches actuelles, est fondé sur de graves erreurs méthodologiques, relevées depuis vingt-cinq ans par un certain nombre de chercheurs (Frenk H. et al., 2000 ; Kumar R. et al., 1977 ; Molimard R., 2001). Ces recherches concernaient la cigarette. Mais aujourd'hui, c'est précisément ce même modèle erroné qui est appliqué aveuglément au narguilé. Ce traitement n'est pas sans rappeler celui de la question des drogues où il a fallu attendre très longtemps avant que l'on ne commence à reconnaître un peu de légitimité à la réduction des risques, voire aux qualités thérapeutiques de certaines drogues illicites.

La recherche contemporaine est principalement le fait de chercheurs états-uniens et moyen-orientaux (y compris égyptiens), publiant en anglais exclusivement et ne prenant en compte que ce qui est publié dans la langue dominante. Et face à une menace déjà qualifiée d'épidémie, la science se fait dans le cadre d'une " coopération internationale " présentée comme " exemplaire » (Maziak 2004b). Malheureusement, elle ne s'embarrasse pas de détails, surtout quand ils sont ethnologiques.

L'émergence inattendue du narguilé, perçu comme « arabe » ou « oriental », dans un $\mathrm{xxI}^{\mathrm{e}}$ siècle états-unien, déroute autant les observateurs individuels que des équipes entières d'experts réunies par l'OMS (OMS.1). Bref, la dimension sanitaire est devenue, ces derniers temps, la question mère. Or, le narguilé suscite des interrogations plus nombreuses et complexes que ne le soupçonnent les observateurs.

4. Petits cigares aromatisés de forme conique constitués d'une petite quantité de tabac enveloppés dans la feuille d'une plante (tendu). L'Inde les produit et les exporte en masse. 
Pourquoi ce développement soudain sur les deux rives de la Méditerranée ? Le narguilé n'est pas seulement un objet matériel inerte ou un « artefact patrimonial » (Bromberger) qui se réduirait à un concentré d'exotisme et d'orientalisme. Il est aussi une pratique. Pour simplifier, les touristes européens sont revenus, de leurs vacances en Égypte, en Turquie ou en Tunisie, avec un narguilé dans leurs valises et, à leur retour, ils ont trouvé des immigrés, particulièrement égyptiens, qui ont ouvert des cafés comme les cafés de chez eux, c'est-à-dire de vrais « cafés d'Orient » (Desmet-Grégoire), à la différence des " cafés à l'européenne », bref des cafés avec des narguilés. Il y a eu rencontre, découverte ou redécouverte $\mathrm{d}^{\prime}$ un usage et nous avons assisté alors à un effet « boule de neige ».

Des hypothèses ont été proposées, comme ce "scénario » d'une équipe syro-états-unienne (Rastam) selon lequel l'émergence des chaînes de télévision par satellite en aurait popularisé l'usage (I'étude se limite au cas du MoyenOrient). Or, si la télévision peut avoir joué un rôle, il s'agit avant tout de la puissante industrie cinématographique égyptienne qui a montré des fumeurs de narguilé, non seulement depuis quelques années mais depuis des décennies, à tous les téléspectateurs du monde arabe. Par conséquent, les Égyptiens sont intervenus à deux niveaux : d'une part, par le chassé-croisé entre les flux touristiques et migratoires liés à la récente mondialisation ; et, d'autre part, par la télévision, si influente dans le monde arabe (Chaouachi, 2000).

\section{ORIGINE DU NARGUILÉ}

L'histoire de l'objet demeure mystérieuse et remonte au-delà de l'introduction du tabac et des instruments diffusés par les Européens aux xvI et xvII siècles. Le narguilé ne serait pas, comme l'histoire officielle européenne du tabac le voudrait,
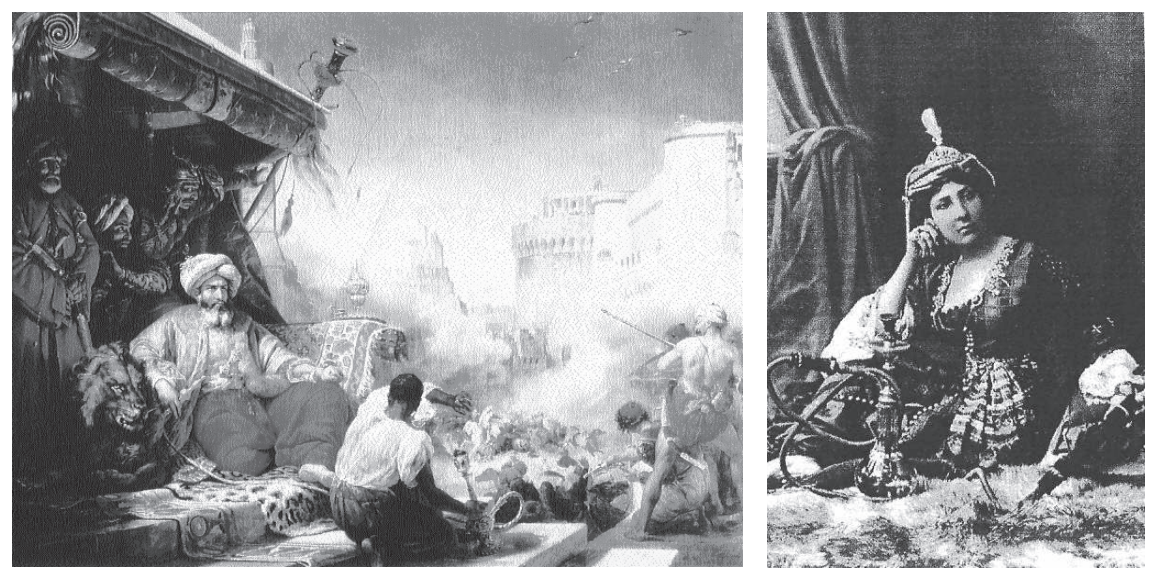

À gauche : Mohammed Ali. À droite : la princesse Chivekiar, petite fille de Ahmet Rifaat Pacha, épouse du prince Fouad, futur roi Fouad

Source : "Mehemet Ali Le Grand », livre écrit par le prince Osman Ibrahim, Paris, Maisonneuve et Larose, 2005 
une adaptation de la pipe courte et coudée qu'ils utilisent encore de nos jours. L'origine du narguilé est revendiquée aujourd'hui par de nombreux peuples: Indiens, Turcs, Arabes, Iraniens, et les Africains auraient aussi de bonnes raisons également de le faire. Tous sont prompts à revendiquer la paternité d'un objet aussi original. Un rapport de l'OMS s'évertue à vouloir définir, dans une tentative de pulvériser un mythe par un autre, une origine indienne (OMS.1). Un médecin d'origine iranienne aurait inventé ce dispositif alors qu'il était en fonction auprès de l'empereur Akbar dans I'Inde mongole de l'an 1605. L'hypothèse la plus plausible pointe vers une origine sud ou est-africaine (Chaouachi, 2000).

\section{Aspects sociaux et culturels : religion, miel et femmes}

Quel rapport le narguilé entretient-il avec la religion ? Les croyants des trois religions monothéistes de la région ont, des siècles durant, fumé et fument encore aujourd'hui le narguilé. Le parcours de la mosquée au café et vice-versa a constitué pendant très longtemps un itinéraire banal de la vie quotidienne. À une époque moins puritaine qu'aujourd'hui, il n'était pas rare de rencontrer dans les cafés des hommes de religion et en particulier des soufis. Quant aux festivités annuelles accompagnant le jeûne rituel, très largement observé dans un pays comme l'Égypte, elles ont fait du narguilé, et de nos jours en font encore, une vedette de la sociabilité arabo-islamique (Chaouachi, 2000).

La convivialité du narguilé s'intensifie à l'occasion des longues nuits du Ramadan. Durant cette période, la commensalité de la «table servie » est aussi celle du narguilé qui suit naturellement le copieux repas du crépuscule. Chaque année, une trentaine de nuits durant, pratiquement tous les cafés, y compris ceux des grands hôtels, proposent des animations diverses - orchestres, pâtisseries, sucreries, boissons - qui se mettent en place à l'heure de la rupture du jeûne et se prolongent souvent jusqu'à l'aube. Le décor est parfois féerique et reproduit, en Égypte comme en Jordanie et d'autres pays encore, I'ambiance d'une tente immense en référence à la vie bédouine. Tous les murs et parois sont recouverts de tapis ou de tissus brodés ou imprimés d'arabesques de couleur rouge, jaune, blanche ou noire. Ces salons ainsi habillés sont appelés, un mois durant, des tentes (khiyâm [ramadâniyya]) et les établissements ne manquent pas de diffuser de la publicité dans les journaux locaux.

En Égypte, des études épidémiologiques ont cherché à analyser l'effet des fatwas les concernant sur l'esprit des amateurs de tabac, narguilé compris (Radwan, 2003). Les intéressés seraient en général au courant de ces fatwas mais peu enclins à changer d'attitude. D'autre part, puisque le mélange est à base de mélasse ou de miel (jusqu'à 70 \%), on peut se demander quelle place cette substance occupe dans la culture locale. Si I'on se réfère à des travaux anthropologiques en Amérique du Sud, « le tabac fumé offre une affinité avec le feu et le miel dilué avec l'eau » (Lévi-Strauss 1967). On est ici en présence d'un phénomène inédit : lavage de la fumée d'un mélange miel-tabac 
(tabamel). Par ailleurs, la qualité thérapeutique du miel est mentionnée clairement dans le Coran (sourate des Abeilles : XVI, 68-69).

Le caractère « égalitaire » du narguilé tant du point de vue social que religieux a beaucoup frappé les voyageurs européens des siècles passés. Claude Lévi-Strauss (1955) observait que "la fraternité islamique repose sur une base culturelle et religieuse. Elle n'a aucun caractère économique ou social. Puisque nous avons le même dieu, le bon musulman sera celui qui partagera son hookah avec le balayeur ». Ce dépassement ou cette ignorance des frontières sociales à propos du narguilé a donné à certains l'illusion de sociétés "sans classes », particulièrement dans l'Empire ottoman (Desmet-Grégoire).

Même homogénéisation pour les générations différentes qui s’y adonnent indistinctement. Il est en effet frappant, en observant les profils de fumeurs dans un " coffee shop » égyptien, jordanien ou libanais, de trouver aussi bien des jeunes gens que des vieillards et de jeunes touristes européennes côtoient des hommes âgés du terroir.

Enfin, s'agissant de la différenciation sexuelle de l'usage, fumer le narguilé ne relève pas, contrairement à un stéréotype ancien, exclusivement du domaine masculin, sauf dans des pays comme la Tunisie et la Libye, pour diverses raisons (Chaouachi, 2000). En Syrie, des jeunes reproduisent les rites des " anciens », moins comme conséquence d'une transmission et d'un apprentissage local que par imitation des touristes venant de loin goûter à leur quotidien local (ibid.). Une équipe syro-états-unienne de chercheurs a rendu les femmes (arabes) responsables de l'épidémie en évoquant la question du «tabou » des cigarettes (Maziak 2005), affirmation bien hâtive. Routh rappelle qu'aux Etats-Unis, dans les années 1920, I'usage du tabac était encore un male domain avant que le cinéma contribue à changer les attitudes. En France, les cafés populaires sont restés longtemps fréquentés majoritairement par les hommes. Un journal français suggère de libérer les femmes musulmanes au moyen de... la cigarette. L'illustration de la couverture représente une jeune femme mi-voilée tenant une cigarette. Le commentaire qui l'accompagne dit: "Monde musulman : les femmes s'affirment $»^{5}$. Les revendications des féministes turques des débuts de la république ont été récemment prises à contre-pied par leurs collègues jordaniennes qui sont allées jusqu'à dire que l'usage du narguilé par les femmes est inacceptable... ${ }^{6} \mathrm{Et}$ que dire des parlementaires égyptiens qui voulaient, il y a deux ans, interdire la chicha aux femmes uniquement? Bref, le narguilé révèle comment les femmes arabes ou musulmanes sont prises entre deux feux : tant celui des intégristes « occidentaux » $\mathrm{qu}^{\prime}$ « orientaux » et leurs relais respectifs de part et d'autre.

5. Courrier international, 679, 6 novembre 2003, www.courrierinternational.com/ hebdo/sommaire.asp?obj_id=159

6. Taha Salwâ, "Nidâ' ilâ chabâbinâ li-tarki al-dukhân wa-l-narjîla » (Appel solennel adressé à nos jeunes pour qu'ils cessent de fumer le narguilé), Amman, quotidien Al-Ra'î, 7 sept. 2000, p. 35 du deuxième cahier. 


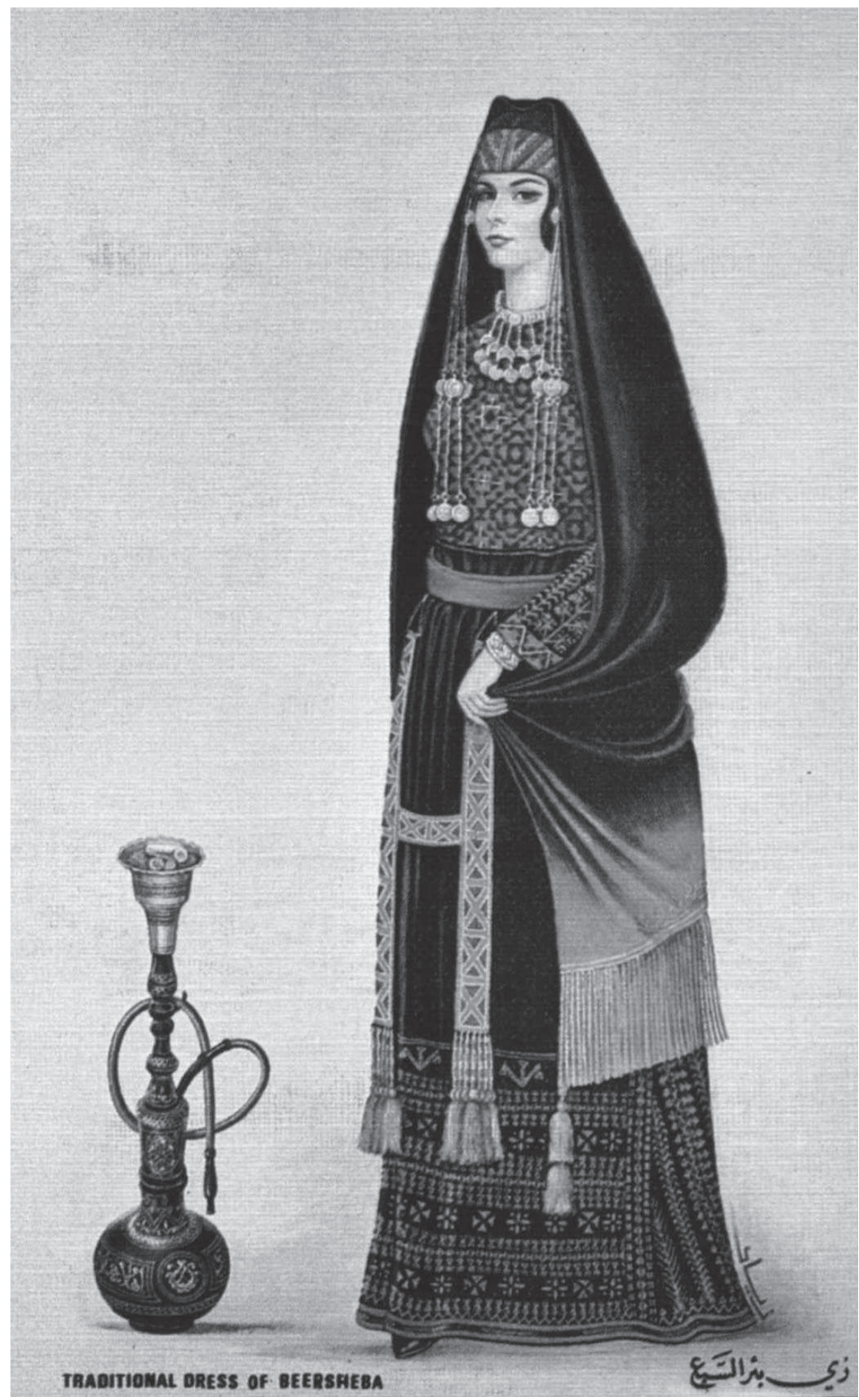

Le narguilé et les femmes en Palestine 


\section{Rapport avec l'identité}

Certains observateurs de terrain ont cru dénoter dans l'usage massif du narguilé dans les sociétés contemporaines, de la Méditerranée orientale notamment, une forme de "nationalisme ». Le rapport d'un consultant pour le bureau responsable du Moyen-Orient de l'OMS, à Alexandrie (Égypte), annonçait ainsi, dans les années 1990, que la pratique du narguilé était maintenant répandue à travers tout le monde arabe et qu'il fallait chercher les raisons de son utilisation dans un mélange d'économie et de sentiments nationalistes (OMS.2). À la lumière de la vogue soudaine et mondiale de cet usage, que dire ? Que les fumeuses et fumeurs, de Honolulu à Novgorod et de Stockholm à Durban, sont des nationalistes? Peut-on rendre les migrants du Moyen-Orient responsables de l'épidémie (Knishkowy, 2005) et y voir un " objet identitaire " pour les diasporas du Moyen-Orient? Pour les uns, opprimés ou dont le pays est occupé, comme les Palestiniens, les Afghans ou les Irakiens, ou encore dans leur pays d'émigration, le narguilé rappelle-t-il à leur mémoire l'art de vivre d'avant une modernité synonyme de défaite?

Les boîtes de tabamel ont été mobilisées pour diffuser une imagerie populaire de cette identité collective. On y voit des figures souvent naïves représentant de vaillants sultans (Al-sultân) et même des pharaons ('arûs al-nîl), le Vieux du village (Chaykh al-baled), des jardins andalous (Al-Andalus), voire d'Eden, et partout présente la convivialité (Layâly-I-uns en référence aussi à la célèbre chanson d'Asmahân) (Chaouachi 2000). En fait, sa pratique apparaît plus comme le surgissement d'une nouvelle culture populaire (De Certeau) que la nouvelle mondialisation des échanges a tendance à refouler. Pour les immigrés indiens, arabes ou autres, le narguilé représente souvent soit une nostalgie d'un pays quitté, soit une forme de résistance aux valeurs actuelles.

$L^{\prime}$ " épidémie » du narguilé révélerait ainsi une forme de néo-orientalisme, mais nourrie par les "Orientaux » eux-mêmes, qui, à travers leurs cafés-narguilés, vendent de la convivialité, du rêve et de l'exotisme aux sociétés de loisirs rongées par l'individualisme où ils se sont installés (Chaouachi, 2006a).

\section{La relation du narguilé avec les drogues}

Récemment, une étude biomédicale alarmiste, venue d'Israël, mettait en garde contre l'usage de drogues dans le narguilé par les adolescents (Knishkowy, 2005). Dans un pays comme l'Égypte, l'usage d'une drogue comme le cannabis est plus complexe qu'ailleurs, en raison notamment de l'acceptation sociale dont elle a fait l'objet pendant très longtemps. Le professeur Fouad alBelasy, de l'université de Mansûra, est l'auteur d'une étude sur la chicha. II a récemment révélé que son père tenait un célèbre coffee shop et qu'il connaît donc bien le monde de la chicha, de la gûza et « ce que l'on met dedans ». Sa description est en effet très claire (al-Belasy, 2006). Ailleurs, un anthropologue 
montre que "fumer le haschich dans une pipe à eau, comme une chanson populaire le fait observer, a pour fonction de "regrouper » les «bien-aimés ». [...] Une séance de consommation de haschich rassemble des gens de diverses professions, classes et de niveaux d'éducation différents, qui se rencontrent dans une atmosphère de fraternité, d'égalité et de liberté vis-à-vis des normes sociales qui prescrivent certains comportements. En outre, ces sessions obéissent à des rituels particuliers »(Khalifa, 1975). Les autorités ont essayé de dé-normaliser I'usage de cette drogue mais d'une manière maladroite qui s'est traduite par une grande confusion entre, d'un côté, des produits réputés "durs » (héroïne, cocaïne, etc.) et, de l'autre, le haschich (Roussillon, 1986).

La relation, imaginaire ou réelle, du narguilé avec les drogues est ancienne. Mais aujourd'hui ce que les usagers consomment n'est que du tabamel, une préparation à base de $30 \%$ et moins de tabac... En Égypte, les usagers de drogues savent parfaitement que le narguilé de type chicha n'est pas très efficace pour extraire le principe actif du cannabis, et des études pharmacologiques l'ont confirmé. Pour cela la petite gûza est plus efficace que la moderne chicha.

\section{La dimension sanitaire : la mère des questions}

Enfin, la nouveauté concerne l'aspect sanitaire et la volonté désormais affirmée des autorités dans le domaine de comparer les effets liés à l'usage du narguilé à ceux de la cigarette.

Cette question ne s'était pas posée pendant des siècles, quand la cigarette $\mathrm{n}^{\prime}$ existait pas encore, et tout au long du siècle passé, où elle coexistait avec un narguilé replié sur l'Afrique et l'Asie. Or, il s'agit de deux mondes différents. La dépendance, rare, existe et toucherait plutôt les ex-usagers de cigarettes décidant, par automédication, de chercher à fumer à moindre risque. A priori, le narguilé n'est pas un tremplin vers la cigarette. Les différences sont grandes: températures $\left(900^{\circ} \mathrm{C}\right.$ contre $\left.100^{\circ} \mathrm{C}\right)$; parcours de la fumée (10 centimètres contre 2 mètres); filtres (acétate de cellulose contre eau) ; temps (5 minutes contre 1 heure) ; usages (individuel contre partagé) ; culture, etc. Ces différences ont des conséquences immenses et insoupçonnées sur la nature des pathologies potentiellement associées. Par exemple, la faible prévalence du cancer du poumon chez les fumeurs de narguilé serait en rapport avec les basses températures en jeu (Rakower, 1962). En effet, les goudrons sont d'autant plus cancérigènes que la température à laquelle ils sont produits est élevée. L'eau du récipient semble être efficace pour retenir, entre autres, et en grande partie, le benzopyrène, un hydrocarbure très dangereux, etc. Enfin, une séance de narguilé ne fournit, dans le meilleur des cas, que l'équivalent en nicotine d'une cigarette. Dans ces conditions, la dépendance à la nicotine est très relative. Elle dépend d'ailleurs des types de tabac (tumbâk, jurâk, tabamel) et du profil et de la carrière, récente et lointaine, des fumeurs. 


\section{L’orientalisme biomédical}

Une certaine forme d'orientalisme a pu dévoyer les sciences humaines et sociales (Saïd, 1980). Les chercheurs en biomédecine n'en sont apparemment pas conscients, qui monopolisent actuellement le débat sur le narguilé. Une étude affiche une photo en couleur du fameux tableau de Delacroix intitulé Femmes $d^{\prime}$ Alger avec une légende qui indique que le narguilé pourrait bien avoir des racines culturelles dans la région : "The social character of waterpipe smoking and acceptability of its use by women may have their roots back in the culture of this region " (Maziak et al. 2005).

L'un des auteurs d'une de ces études déclare que les femmes du MoyenOrient s'adonnent davantage au narguilé et que cette pratique serait plus acceptable parce que liée à des activités traditionnelles plutôt qu'à des activités occidentales $(s i c) .{ }^{7}$ Ce type de déclaration fait le va-et-vient entre les journaux biomédicaux scientifiques et les médias états-uniens sans parler des agences de presse internationales. Or le renouveau de la pratique parmi les femmes arabes n'est pas lié à des tâches domestiques mais, au contraire, à des activités bien « modernes», comme celle de sortir dans les cafés, comme les hommes. Non pas aller dans les fameux "Cafés d'Orient " traditionnellement réservés aux hommes (Desmet-Grégoire, Georgeon), mais dans ces nouveaux cafés néoorientalistes, en Orient même et en dehors de cette région, ouverts à tous, jeunes et moins jeunes, femmes et hommes, filles et garçons, touristes et gens locaux, etc. Le spectacle qui irritait les parlementaires égyptiens évoqués plus haut est bien celui de ces jeunes filles et femmes s'adonnant au narguilé en public...

Après les hommes migrants du Moyen-Orient, il restait à impliquer les enfants. C'est chose faite avec le rapport de l'OMS (OMS.1) qui affirme qu'il est courant, dans le monde arabe (techniquement nommé «Asie du Sud-Ouest et Afrique du Nord »...), de voir les enfants fumer le narguilé avec leurs parents...

Les équipes mixtes de chercheurs sur ces sujets sont composées majoritairement de chercheurs des États-Unis et du Moyen-Orient qui ont étudié en majorité aux États-Unis ou bien travaillent avec des bourses de ce pays ou encore dans des centres financés par ce dernier. Logiquement, leur idéologie se montre conforme à celle des Etats-Unis, qui souhaitent en finir avec le narguilé. Par conséquent, les meilleures études dans ce domaine ont été conduites par des chercheurs indépendants des pays pauvres qui les ont produites avec peu de moyens (Sajid, Zahran, Macaron, Behera, Shafagoj). Ils ne parlent pas d'emblée de nicotine addiction ni ne se livrent à des comparaisons

7. "Now it's even more accepted for women to smoke water pipes there rather than cigarettes, probably because it's associated with traditional activities rather than Western activities." Thomas Eissenberg, cité dans El-Boghdady Dina, "Hookah Lounges Gaining Fans in District: Proposed Smoking Ban Could Shut Them Down Unless Exemption Is Made", Washington Post (online), 24 juin 2005. 
avec la cigarette, pas plus qu'ils ne versent dans la diabolisation de l'objet. Ils se bornent à décrire des expériences, avec une bonne connaissance de leurs propres sociétés. Le récent projet "Research Assistance Matching " (aide au repérage de partenaires de recherche) a précisément été lancé par les responsables du centre syro-états-unien et de I'Institute for Global Tobacco Control (Johns Hopkins School of Public Health) ${ }^{8}$ dans le but de capter ces expertises nouvelles.

\section{Des infrastructures pour combattre la menace}

L'usage massif de la chicha a en effet très vite inquiété les responsables de la santé publique, particulièrement aux États-Unis. Des centres de surveillance socio-sanitaire, entièrement financés par les États-Unis d'Amérique, voire le Canada, et comprenant des équipes biomédicales mixtes de spécialistes, se sont installés en Égypte, en Syrie et au Liban. Pour la première fois dans son histoire, en 2005, l'Organisation mondiale de la santé (OMS.1) a publié un rapport d'experts sur ce sujet. Or, ce document, qui s'étonne du manque d'études, comprend de nombreuses erreurs méthodologiques, par méconnaissance des cultures et des sociétés concernées (Chaouachi 2006b). Comme dans le domaine de la prévention de l'abus des drogues, I'anthropologie est souvent perçue comme une forme d'apologie indésirable par les tenants du paradigme dominant quant au lien santé-société. Elle est reléguée dans le folklore, au mieux à l'ethnographie. Le bureau régional de l'OMS en Égypte a même organisé en 2002, en collaboration avec le Forum arabe des sciences sociales et médicales, une réunion regroupant 30 participants venus de 16 pays en vue de lancer une étude-pilote sur l'usage du narguilé dans six pays de la région. Le financement serait en cours.

Dans le cas de la Syrie, le SCTS (Syrian Center for Tobacco Studies) ${ }^{9}$ est financé pour cinq ans par les puissants National Institutes of Health (ÉtatsUnis). C'est un partenariat regroupant, en dehors de chercheurs syriens, I'Université de Memphis et celle de Virginia Commonwealth. Ses études se sont distinguées par une attention exagérée portée au narguilé. La grande majorité de la production scientifique ne porte pas sur le danger des cigarettes. Les Syriens fument des cigarettes venant d'Amérique du Nord dont les intérêts sont en jeu puisque nous sommes dans le "tiers-monde ", une région vers laquelle les cigarettiers du monde se sont stratégiquement repliés, suite à l'action des groupes anti-tabac en Europe et en Amérique du Nord notamment. Ils consomment aussi des cigarettes nationales (le fameux tabac

8. "Research Assistance Matching ». Voir tc.bmjjournals.com/cgi/content/extract/ $14 / 3 / 151$

9. Syrian Center for Tobacco Studies (www.scts-sy.org). 
de Lattaquié, entre autres) dont les intérêts directs sont ceux de l'État syrien. Bref, ce centre n'a pratiquement publié, depuis plus de deux ans, que des études sur le narguilé...

Au Liban, I'acteur clé est l'American University of Beirut dont les études sont financées par le conseil d'administration de l'université et le RITC (Research for International Tobacco Control) canadien. Ses études se sont distinguées aussi par une attention intense sur le narguilé teintée d'une diabolisation prononcée. Son meilleur produit est une double étude (Shihadeh) à partir d'une machine à fumer en laboratoire, censée reproduire le comportement du fumeur de narguilé. Ces deux études, qui font référence, y compris dans le rapport de I'OMS (dont l'un des deux auteurs est Shihadeh lui-même), et sont citées partout, reposent sur une aberration méthodologique. Elles ne tiennent pas compte du caractère sporadique de la consommation du narguilé ni encore de la manipulation du charbon et de sa nature (Chaouachi, 2007).

En Égypte, le centre ESPRI (Egyptian Smoking Prevention Research Institute) a pour objectif officiel de lutter contre le tabagisme. Il s'agit d'un projet financé pour cinq ans encore une fois par les National Institutes of Health (États-Unis). II opère conjointement entre I'Université du Maryland (Baltimore) et le ministère égyptien de la Santé et de la Population, I'Université de Georgetown (Washington DC), celles de 'Ayyn Chams et du Caire. Ses locaux se trouvent à I'Institut national de recherche en hépatologie et médecine tropicale situé au Caire. Il a établi des liens avec l'OMS, le syndicat médical égyptien, l'association égyptienne des pneumologues et diverses ONG. Le centre a publié une dizaine d'études dans le Journal of the Egyptian Society of Parasitology.

\section{Modèle virologique}

La visée des centres syrien et libanais est ouvertement éradicatrice vis-à-vis du narguilé. L'un des chercheurs du centre syro-états-unien déclarait ouvertement que le but est de tuer l'épidémie dans l'œuf (nip it in the bud). Ces réactions sont d'autant plus étranges que le narguilé $n$ 'est pas apparu soudainement ex-nihilo. Il aurait fallu s'y prendre quatre cents ans auparavant. Le chercheur affirme également que les Moyen-Orientaux se seraient fait du mal pendant des siècles, comme s'il disposait d'études épidémiologiques rétrospectives pour les siècles passés...

Ainsi, la réponse au péril annoncé semble relever d'un modèle virologique mais le virus ne se trouve pas seulement en Égypte. Il a déjà touché le territoire américain, où des centaines de bars à chicha ont soudain ouvert leurs portes et devant lesquels se bousculent les clients. Comment faire pour extirper ce virus? Les responsables commencent à constater que l'affaire est complexe et comporte apparemment un très important versant socioculturel. 


\section{Production des centres de surveillance}

Devant une " épidémie » dont l'origine est inconnue, un scénario s'est mis en place. Des revues alarmistes de la littérature existante avertissent que le narguilé serait un facteur de risque pour des maladies comme la tuberculose, I'hépatite C, l'infection par Helicobacter pylori, ${ }^{10}$ et même l'aspergillose. Un chercheur rapporte le cas unique, évidemment anecdotique, d'un individu immunodéprimé atteint d'aspergillose pulmonaire invasive à la suite de son utilisation d'un narguilé chargé d'un mélange de marijuana et de tabac contaminé par Aspergillus. Un détail frappe de surcroît : l'étude rapporte que l'eau du vase n'avait pas été changée pendant plusieurs semaines... Or, l'on observe dans les cafés - ce qui est évident pour un Égyptien mais pas pour un Canadien ou un Italien - que le récipient est systématiquement lavé et rempli à nouveau d'eau propre à la fin de chaque séance, soit plusieurs fois par jour (Chaouachi 2006c).

\section{Le modèle des drogues : exemple égyptien}

En Égypte, une personnalité éminente sur la scène politico-médiatique, Ibrâhîm Nâfi ${ }^{\prime 11}$, entend traiter exhaustivement de la catastrophe que représente pour elle l'usage de drogues dans le pays. Toutes les substances sont passées en revue mais rien dans le texte ne traite du narguilé, si ce n'est deux caricatures, I'une montrant un groupe de gens qui tous se droguent de diverses manières, entre autres par voie intraveineuse et au moyen de la gûza. Sur l'autre dessin, qui représente un couple, les prunelles des yeux de la femme qui sourit sont deux gûza. Quant au mari, qui compte son argent, c'est son nez qui prend la forme de la célèbre pipe à eau égyptienne...

Dans un autre ouvrage, le même auteur ${ }^{12}$ aborde notre objet dans un paragraphe intitulé « la chicha, la gûza et la pipe à eau, comme on l'appelle en Amérique " $(s i c)$. Ces instruments sont présentés comme adaptés à la consommation de marijuana et de haschich. En évoquant le contexte juridique de la loi fédérale de 1986, Ibrâhîm Nâfi' semble oublier que le lecteur auquel il s'adresse est égyptien, ou en tout cas arabophone, et qu'il ne se

10. Cette bactérie peut provoquer un ulcère duodénal, maladie longtemps considérée comme d'origine psychosomatique, mais aussi des gastrites et certains cancers gastriques.

11. I. Nâfi', 1989, Kârithat-l-idmân (La catastrophe de la dépendance), Le Caire, Markaz al-Ahrâm li-I-tarjama wa-I-nachr, pp. 15 et 129.

12. I. Nâfi', 1991, Fî baytinâ mudmin - Kayfa namna' al-kâritha? (Il y a des personnes dépendantes chez nous - que faire pour empêcher la catastrophe?), Le Caire, Markaz al-Ahrâm li-l-tarjama wa-l-nachr, pp. 61-64 et 73-74. 
trouve pas aux États-Unis... Cette loi, poursuit I'auteur, interdit la fabrication et l'utilisation d'instruments de consommation de drogues : les drug paraphernalia. Les parents, insiste-til, peuvent s'attendre à trouver "un nombre croissant de pipes «maison» chez eux »! Le rédacteur en chef du plus grand quotidien égyptien, $A /$ Ahrâm, évite apparemment de parler de son pays. En effet, les pipes à eau sont partout, dans tous les domiciles et tous les cafés. Son discours, qui est celui des officiels américains, constitue donc une importation pure et simple. Le résultat est la confusion la plus totale (Roussillon, 1987).

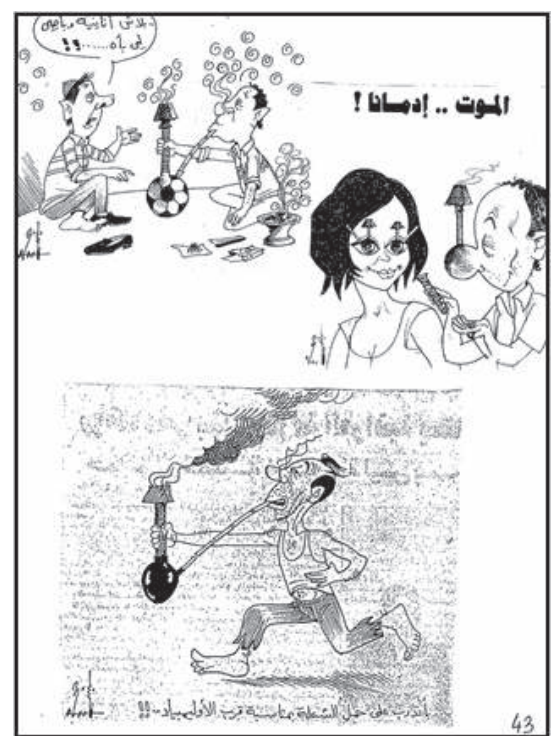

Égypte : le narguilé entre le diable et la tradition

\section{CONFUSION}

Durant les trois dernières années qui ont suivi la «prise de conscience » de l'ampleur du phénomène (Congrès mondial sur le tabac, Helsinki, 2003), on a assisté à une confusion croissante dans les études biomédicales. Ces errements auraient été évités si la volonté de collaborer entre représentants des sciences sociales et biomédicales, indépendamment de leurs affiliations, de leur langue et de leurs travaux respectifs, avait prévalu. Ce ne fut pas le cas. Une fameuse publication (Maziak 2004), qui a par la suite servi de base au rapport de l'OMS, affirme qu'une étude établit le lien entre l'usage du narguilé et le cancer du poumon. ${ }^{13}$

La très grande majorité des études ignorent le profil des volontaires qui se sont prêtés à l'expérience. Le plus souvent, il s'agissait de fumeurs de cigarettes s'adonnant occasionnellement au narguilé et d'ex-fumeurs de cigarettes s'abstenant depuis un certain temps qui se mettent au narguilé. Or le corps garde une mémoire physiologique et comportementale des pratiques tabagiques. Par exemple, un ex-fumeur de cigarettes a tendance à inhaler profondément dans un effort pour obtenir le même effet que celui dû aux alcaloïdes.

13. Maziak W, Ward KD, Afifi Soweid RA, Eissenberg T., 2004, "Tobacco smoking using a waterpipe: a re-emerging strain in a global epidemic", Tobacco Control, 13: 327-333. Voir notre analyse critique de cette étude dans Chaouachi (Kamal), "Letter to the Editor: Serious Errors in this Study", Tobacco Control; 2 Dec. 2004. tc.bmjjournals. com/cgi/eletters/13/4/327. 
Jusqu'à ce jour, les questionnaires utilisés dans les enquêtes épidémiologiques n'en font pas mention. Ainsi, une chercheuse au Liban prétend sonder le degré de connaissances qu'auraient les individus interrogés en se basant sur des questions relatives à des faits qui n'ont pas été établis avec la rigueur scientifique nécessaire (Chaaya, 2004). Une telle étude comprend un tableau statistique sur la «justesse » des réponses données par des étudiants à des questions comme : "L'usage du narguilé a un lien avec le cancer de la cavité orale : vrai ou faux ? ", "L'usage du narguilé a un lien avec l'apparition d'ulcères : vrai ou faux?», ou encore "Le narguilé contient des métaux lourds : vrai ou faux?». De telles études ne permettent nullement de conclure à la responsabilité, même indirecte, de l'usage du narguilé dans les pathologies en question. Quant aux "métaux lourds», l'étude en rapport faisait appel à un type particulier de charbon dont la nature n'a pas été précisée aux enquêtés et qui n'est pas toujours celui qu'ils se représentent.

Les auteurs de nombre d'études considèrent ces faits comme définitifs et établis et les citent sans les accompagner de commentaires pertinents. Aucune distinction n'est faite entre les divers types de tabac, de préparations à base de tabac (tabamel, tumbâk, jurâk), entre les divers types de narguilé. Par exemple, la gûza égyptienne par sa taille est très différente d'un imposant hookah indien ou d'une madâ'a yéménite ou d'un narguilé chinois.

Une étude libanaise menée sur un prototype artificiel de chicha en laboratoire à l'American University of Beirut et qui sert de modèle à des centaines d'associations contre le cancer et autres organismes de prévention à travers le monde, sans oublier tous les ministères de la Santé du monde, se fonde en fait sur des mesures où 171 bouffées ont été prélevées, chacune d'un volume de 0,53 litre et durant 2,6 secondes toutes les 17 secondes; soit au total environ une heure. En dehors de ces paramètres volontairement exagérés et différant fortement de la réalité telle qu'elle se présente dans un café du Caire par exemple, une quantité réduite de tabamel (10 grammes) a été utilisée et le charbon n'a pas été changé de place. Les conditions ont ainsi été réunies pour contraindre le tabamel à se carboniser et produire des taux inquiétants de goudrons entre autres, détail d'importance dans lequel réside le premier biais majeur de cette étude pourtant mondialement célèbre. Le second biais majeur tient au fait que la fumée fut absorbée de manière périodique, comme un métronome en se basant sur une «moyenne » de chiffres observée dans la réalité. Or, faire une moyenne sur une durée d'une heure est une aberration méthodologique. En effet, de multiples micro et macroévénements se produisent en une heure. Par exemple, le fumeur pose le tuyau, boit du café, passe le tuyau à une autre personne, parle, etc.

L'application du dogme de la « dépendance à la nicotine » (Frenk) conduit à diaboliser encore le narguilé en le présentant comme un tremplin (gateway) vers l'usage de cigarettes. 


\section{Le problème de la langue}

Les auteurs arabophones non anglophones sont restés jusqu'à présent silencieux bien qu'ils aient "des choses à dire ». Le monde des publications dans le domaine des sciences biomédicales est, par ailleurs, très centralisé, autour d'une base de données internationale comme Medline. C'est un cercle vicieux puisque les références bibliographiques citées sont en anglais dans $95 \%$ des cas. Rappelons au passage que le Nida (National Institute of Drug Abuse) finance à lui seul $80 \%$ de la recherche mondiale sur les addictions (drogues, tabac).

\section{CONCLUSION}

Presque universellement transplantée, la pratique du narguilé émerge dans la modernité dans le prolongement d'une tradition vieille de quatre siècles. L'objet est changeant, circulant, chargé d'oppositions. Exprimant le surgissement d'une culture populaire, il n'est pas un produit mais un contre-produit, social et culturel de la nouvelle mondialisation. Par ailleurs, son émergence soudaine prend aussi la forme d'un boomerang voire d'une farce particulièrement jouée à l'adresse de l'anthropologie officielle.

Relevant d'un usage traditionnel et sporadique de produits à base de tabac, le narguilé n'a apparemment pas posé de problème sanitaire majeur au cours des siècles passés. Il en pose apparemment beaucoup aujourd'hui... J'ai montré comment, dans le cadre d'une coopération internationale entre les États-Unis et le Moyen-Orient, la science se fabrique à propos d'un problème potentiel de santé publique. Cette fabrication, qui se fait dans I'urgence et la confusion, donne à voir les relations entre logiques de pouvoir et logiques de production scientifique. Un tel problème, désormais appelé " épidémie », requiert en fait un programme interdisciplinaire de recherches afin d'apporter l'équilibre nécessaire dans le traitement de la question accaparée par une vision excessivement positiviste et néo-orientaliste. Premièrement, il s'agit de produire une analyse nuancée grâce à l'apport des sciences sociales et humaines sur la question, contre toute approche exclusivement biomédicale. Deuxièmement, d'un point de vue linguistique, la recherche francophone dans ce domaine doit avoir un droit de parole face à une littérature hégémonique de langue anglaise.

Un autre discours a raison d'être à côté d'une "vision » inféodée à un modèle à visée clairement éradicatrice. Malgré l'hostilité de l'environnement géopolitique actuel, seule une recherche objective et dépassionnée sur cette question permettra d'écrire en Égypte et ailleurs un chapitre exemplaire sur la manière dont la science moderne doit aborder les défis socio-sanitaires, en permettant, par la même occasion, un rééquilibrage nécessaire. 


\section{RÉFÉRENCES BIBLIOGRAPHIQUES}

Al-Belasy F., 2006, "To the Editor: A Personal Experience with Goza and Shisha Smoking », Tobacco Control (12 June), tc.bmj.com/cgi/eletters/15/ suppl_1/i24\#552

BOUVIer Pierre, 1995, Socio-anthropologie du contemporain, Paris, Galilée.

Bromberger C., 2001, in Albera D., Blok. Et Bromberger C. (éds.), Anthropology of the Mediterranean (L'anthropologie de la Méditerranée), Maisonneuve et Larose, Maison méditerranéenne des sciences de l'homme, p. 617-620. Certeau (de) Michel, Luce Giard et Pierre Mayol, 1994, L'Invention du quotidien : habiter, cuisiner, Paris, Gallimard.

Chatya M., Z.E. Roueiheb, H. Chemaitelly, G. Azar, J. Nasr et B. Al-Sahab, 2004, "Argileh Smoking Among University Students: A New Tobacco Epidemic », Nicotine \& Tobacco Research (June), 6 (3):457-63.

Chaouachi Kamal, 1997, Le Narguilé. Anthropologie d'un mode d'usage de drogues douces, L'Harmattan, 262 pages.

- 2000, Le Narguilé : analyse socio-anthropologique. Culture, convivialité, histoire et tabacologie d'un mode d'usage populaire du tabac, thèse de doctorat, Université Paris-X, 420 pages, publiée et diffusée par ANRT (Lille, France), www.anrtheses.com.fr.

- 2001, "Le Narguilé au Yémen » in Yémen in D'un itinéraire à l'autre (dir. S. Naim), Paris, Maisonneuve et Larose, pp. 130-147.

- 2002, Le Monde du narguilé, Paris, Maisonneuve et Larose, 156 pages (couleur).

- 2004, "Letter to the Editor: Serious Errors in this Study », Tobacco Control (2 Dec.). A critical analysis of the following study: Maziak W., Ward K.D., Afifi Soweid R.A., Eissenberg T., 2004, "Tobacco smoking using a waterpipe: a re-emerging strain in a global epidemic", Tobacco Control, 13: 327-333 ; tc.bmjjournals.com/cgi/eletters/13/4/327.

- 2004, "Shisha, hookah. Le Narguilé au XXle siècle. Bref état des connaissances scientifiques » [Narghile, Hookah in the 21st Century: An Overview of the Scientific Knowledge], Le Courrier des addictions (octobre), 6 (4): 150-2.

- 2005, "Post-Publication Peer Review: Errors in this New Review », Pediatrics (15 Aug.). A critical analysis of the following study: B. Knishkowy and M. Amitai, 2005, «Waterpipe (Narghile) Smoking: An Emerging Health Risk Behavior », Pediatrics (July) ; 116(1): e113-9 ; pediatrics.aappublications. org/cgi/eletters/116/1/e113.

- 2005a, «Presentazione del narghilè e del suo uso. Guida critica della letteratura scientifica sul narghilè (shisha, hookah, waterpipe). Dalle origini ai giorni nostri : necessità di un approccio interdisciplinare socio-antropologico, medico e farmacologico» [«Revue critique de la littérature scientifique sur le narguilé (chicha, narghilé, hookah, pipe à eau) des origines à nos jours : la nécessité $\mathrm{d}^{\prime}$ une approche transdisciplinaire socioanthropologique, médicale et pharmacologique »], Tabaccologia, 1: 
39-47 (www.tabaccologia.org) et trois numéros suivants : aspects chimiques (2005c, 3: 27-33), pathologiques (2006c, 1:27-34) et socio-sanitaires (automne 2006d).

- 2005b, «The Recent Development of Hookah Use in the World: A Serious Epidemic or Just a Passing Fad? The Need for a Socio-Anthropological and Medical Approach », IFSSH (International Forum for Social Sciences and Health), World Congress "Health Challenges of the Third Millenium », Istanbul, 21-26Aug. Publié par la Yeditepe University, Dept. of Anthropology, 2005b (Aug.), tome I, pp. 360-1.

- 2006a, «Culture matérielle et orientalisme. L'exemple d'une recherche socioanthropologique sur le narguilé », Arabica, tome LIII, 2, pp. 177-209, Koninklijke Brill NV (Leiden) (en ligne à brill.nl).

- 2006b, "A Critique of the WHO's TobReg "Advisory Note" entitled: "Waterpipe Tobacco Smoking: Health Effects, Research Needs and Recommended Actions by Regulators" »[Fumer du tabac au moyen du narguilé : effets sur la santé, besoins en recherche et actions recommandées par les régulateurs] (2005), Journal of Negative Results in Biomedicine, 5:17; www.jnrbm.com/content/5/1/17.

- 2006c, «E-Letter to the Editor: Syria, Lebanon, Tobacco Research in General and Narghile (Hookah, Shisha) Smoking in Particular », Tobacco Control (8 June). A critical analysis of the following study: Ward K.D., Eissenberg T., Rastam S., Asfar T., Mzayek F., Fouad M.F., Hammal F.,Mock J., Maziak W., "The Tobacco Epidemic in Syria", Tobacco Control ; 15 ; 24-29; tc.bmjjournals.com/cgi/eletters/15/suppl_1/i24

- 2006, "Letter to the Editor: Arabs Neither Need a Scientific Revolution Nor Are They a Cultural Exception», Science (e-Letter, 7 March). A critical analysis of the following study: Maziak W., 2005, "Global Voices of Science. Science in the Arab World:Vision of Glories Beyond », Science (June 3) ; 308 (5727): 1416-8; www.sciencemag.org/cgi/eletters/308/5727/1416\#3253

- 2006, "Letter to the Editor: Some Misconceptions in a Good Alert Paper », Tobacco Control (18 January). A critical analysis of the following study: Afifi-Soweid (Rima), 2005, "Lebanon: water pipe line to youth », Tobacco Control, 14:363-4; tc.bmjjournals.com/cgi/eletters/14/6/363-a\#479.

- 2007, The Medical Consequences of Narghile (Hookah, Shisha). Use in the World, Epidemiology and Public Health:3 (sous presse).

Desmet-GréGoire Hélène et François Georgeon (dir.), 1997, Cafés d'Orient revisités, Paris, éd. CNRS Ethnologie.

Frenk Hanan et Reuven Dar, 2000, A Critique of Nicotine Addiction, Boston, Kluwer Academic Publishers.

Ghalwash M. et J. Martin, "Fishawy's Café: Two Centuries of Tea », revue Aramco World, Houston, vol.47, $\mathrm{n}^{\circ} 5$, pp. 2-7.

Khalifa A., 1975, "Traditional Patterns of Hashish Use in Egypt », in World Anthropology: Cannabis and Culture, Mouton Publishers, New York, pp. 195-206. 
KNishkowy B. and Y. Amital, 2005, "Water-Pipe (Narghile) Smoking: An Emerging Health Risk Behavior », Pediatrics, 116(1): e113-9. Lire notre réaction : Chaouachi (Kamal), « Post-publication Peer Review »....

Kumar R., E.C. Cooke, M.H. Lader and M.A. Rusell, 1977, "Is Nicotine Important to Tobacco Smoking? ", Clinical Pharmacology \& Therapeutics (May), 21(5):520-9.

Levi-Strauss Claude, 1955, Tristes tropiques, Paris, Plon.

- 1967, Du miel aux cendres, Paris, Librairie Plon.

Maziak W., T. Eissenberg and K.D. Ward, 2005, "Patterns of Waterpipe Use and

Dependence: Implications for Intervention Development», Pharmacol Biochem Behav. (January), 80(1):173-9.

Maziak W., K.D. Ward, R.A. Afifi Soweid and T. Eissenberg, 2004a, « Tobacco Smoking Using a Waterpipe: a re-Emerging Strain in a Global Epidemic », Tobacco Control, 13: 327-333. Lire notre analyse critique de cette étude : Chaouachi (Kamal), "Letter to the Editor: Serious Errors in this Study »...

Maziak W., K.D. Ward, T. EissenberG, R.C. Klesges and U. Keil, 2004b, "The

Syrian Center for Tobacco Studies: a Model of International Partnership for the Creation of Sustainable Research Capacity in Developing Countries ", Promot Educ., 11(2):93-7, 116, 134.

Molimard R. 2001, "Dépendance, la nicotine est-elle la seule responsable?»,

Soins Psychiatr. (mai-juin), (214):33-5.

Morin Edgar, 1994, La Complexité humaine, Paris, Seuil.

NÂFI' Ibrâhîm, 1989, Kârithat-al-idman (La catastrophe de la dépendance), Le Caire, Markaz al-Ahrâm li-l-tarjama wa-l-nachr, pp. 15 et 129.

- 1991, Fî beytinâ mudmin - Kayfa namna' al-kâritha ? (Il y a une personne dépendante chez nous - que faire pour empêcher la catastrophe ?), Le Caire, Markaz al-Ahrâm li-l-tarjama wa-I-nachr, pp. 61-64 et 73-74.

OMS.1 (Organisation mondiale de la santé), 2005, rapport "Advisory Note: Waterpipe Tobacco Smoking: Health Effects, Research Needs and Recommended Actions by Regulators », publié par le WHO Study Group on Tobacco Product Regulation.

OMS.2, rapport de K. Rothwell, non daté, "Smoking in the Eastern Mediterranean Region», WHO (World Health Organisation) Eastern Mediterranean Regional Office, Alexandrie.

PARAdeise Catherine, 1980, "Sociabilité et Culture de classe », Revue française de sociologie (octobre-décembre), XXI-4, pp. 571-597.

Radwan G.N., E. Israel, M. El-Setouhy, F. Abdel-Aziz, N. Mikhail and M.K. Mohamed, 2003b, "Impact of Religious Rulings (Fatwa) on Smoking », Journal of the Egyptian Society of Parasitology, 33 (3 Suppl.):1087-101.

RAKOWER J. and B. FAtAL, 1962, "Study of Narghile Smoking in Relation to Cancer of the Lung ", BrJ Cancer (March), 16:1-6.

Rastam S., K.D. Ward, T. Eissenberg and W. Maziak, 2004, "Estimating the Beginning of the Waterpipe Epidemic in Syria », BMC Public Health, 4:32. 
Roussillon Alain, 1986, La Lutte contre les stupéfiants en Égypte: enjeux sociaux d'une répression, Le Caire, Cedej-U.A., 1165 CNRS.

Routh H.B., K.R. Bhowmik, J.L. Parish and L.C. Parish, 1998, "Historical Aspects of Tobacco Use and Smoking », Clin. Dermatol. (septembre-octobre), 16(5):539-44.

SAID Edward, 1980, L'Orientalisme : L'Orient créé par l'Occident (titre original : Orientalism, 1978, trad. fr. par Catherine Malamud, préf. De Tzvetan Todorov), Paris, Seuil.

Shimadem A. et R. Salem, 2005, "Food and Chemical Toxicology: Polycyclic Aromatic Hydrocarbons, Carbon Monoxide, "Tar", and Nicotine in the Mainstream Smoke Aerosol of the Narghile Waterpipe », Food and Chemical Toxicology, 43(5): 655-661. 